\title{
European regulatory perspectives for innovative therapies
}

\author{
S. Ormarsdottir $\cdot$ J.-Y. Reginster $\cdot$ E. Abadie
}

Received: 6 December 2007 / Accepted: 21 January 2008 / Published online: 29 March 2008

(C) International Osteoporosis Foundation and National Osteoporosis Foundation 2008

\begin{abstract}
The current regulatory requirements offer accelerated assessment of innovative therapies in Europe. Future perspectives include the need for increased interaction between stakeholders in pharmaceutical development. Development of new, high quality, effective and safe medicines in Europe is the common goal of academia, pharmaceutical industry and regulatory authorities. To achieve this, it is important that regulatory requirements do not hinder innovation and vice versa, innovation cannot be allowed to proceed without concerns for public health. Interaction between stakeholders in pharmaceutical develop-
\end{abstract}

The views presented are those of the individual and may not be understood or quoted as being made on behalf of EMEA or reflecting the position of EMEA.

\author{
S. Ormarsdottir \\ CHMP member, Senior Expert, \\ Icelandic Medicines Control Agency, \\ Seltjarnarnes, Iceland \\ J.-Y. Reginster \\ President, European Society for Clinical and Economic Aspects \\ of Osteoporosis and Osteoarthritis (ESCEO); \\ URL: http://www.iofbonehealth.org/about-iof/member-societies/ \\ society.html?societyID $=193$ \\ J.-Y. Reginster \\ President, Group for the Respect of Ethics and Excellence \\ in Science (GREES); CHMP Expert, \\ Liege, Belgium \\ E. Abadie \\ CHMP chair, EMEA and Scientific Advisor, \\ General Directorate, AFSSAPS, \\ Saint-Denis, France \\ J.-Y. Reginster $(\square)$ \\ Bone and Cartilage Metabolism Research Unit, \\ CHU Centre-Ville, \\ Policliniques L. Brull, Quai Godefroid Kurth 45 (9ème étage), \\ 4020 Liege, Belgium \\ e-mail: jyreginster@ulg.ac.be
}

ment is of the utmost importance. A dialogue has begun and in the future it will be the responsibility of all stakeholders to ensure continuous exchanges in an environment that is characterised by new scientific advances and global development programmes.

Keywords Academia - Drug development . European Medicines Agency - Innovative medicines · Pharmaceutical industry $\cdot$ Regulatory requirements

\section{Introduction}

The European regulatory perspective of innovative therapies can be put into one phrase: "Protection of public health". It has to be ensured that medicines will not bring more harm than benefit, thus according to the well-known principle of all medical treatment "non nocere". At the same time, it is important that the objective of safeguarding public health is obtained by means that do not hinder the development of innovative therapies. The need to have new products on the market fast, especially for life-threatening diseases and where there is an unmet medical need, has been a challenge to improve the approval procedures in Europe. Furthermore, emerging advanced therapies and new technologies challenge the current requirements for new medicinal products. With more complex global development programmes and increasing research and development $(R \& D)$ costs for a new active substance, predictability of outcome is becoming key in development programmes. As a consequence, regulators are increasingly being consulted during drug development, both with respect to scientific issues as well as regulatory issues. This interaction is still evolving and it is foreseen that interaction with all those involved in pharmaceutical development and in the use of medicines will only increase in the coming years. 


\section{European regulatory requirements}

Regulatory involvement in the development of new medicinal products starts already at the stage of clinical trials. The European Union (EU) Clinical Trial Directive implemented in 2004 provides the framework for the requirements and approval procedures of clinical trials in the EU [1]. Approval by national authorities and ethics committees is needed for all clinical trials according to the definition provided by the directive, from Phase I to Phase IV trials. General requirements include acceptable quality of the product, according to the principles of Good Manufacturing Practice [2] and adequate design of the clinical trial according to the principles of Good Clinical Practice [3]. With the Directive, rules concerning inspections of clinical trials by national authorities were eventually set, since in the past there had not been a legal basis for this in all European countries. Ethics follow the principles laid down in the Helsinki declaration from 1996, which has been a source of debate as the latest version of the Helsinki declaration was not followed. Accordingly, comparison of a new test drug with the best current treatment as laid down in the latest version of the Helsinki declaration is not required; however, this does not mean that there is a general acceptance of placebo controlled trials in every situation by European regulatory authorities or ethics committees. The Clinical Trial Directive has meant improvements in the time frames for approving clinical trials within the union; however, the problem of diverging views and requirements for multi-center trials submitted to the various national authorities remains and the question has been raised whether a "centralised procedure" for approval of clinical trials should be introduced to reduce this disharmony. Furthermore, the Directive has been criticised for tough general requirements, especially the requirements concerning the quality of an investigational product which have made it more difficult for academia to initiate clinical trials without the involvement of pharmaceutical industry [4]. The feeling among academia was that they had been left out of the discussion during the preparation of the Directive and the overall frustration among academia can be seen as a reminder for future interactions when it comes to drug development.

Once clinical trials are finalised, the results are submitted to regulatory authorities for approval for marketing authorisation. The benefit/risk balance (efficacy versus safety) of the new medicinal product is assessed based on science and importantly, irrespective of economic aspects. In general, results from Phase II and III trials form the basis for support of clinical efficacy and safety of a new active substance. Phase III trials are intended to mimic the actual use of the product as much as possible and for this purpose. Phase III trials have to be of sufficient size, performed in the relevant population using appropriate comparators and relevant clinical endpoints. The results with regard to efficacy have to be statistically significant, but also clinically meaningful. A product with doubtful efficacy can also be seen as a safety and as an ethical issue, e.g., by delaying appropriate patients management with more effective treatments. Conversely it is a common misunderstanding that, for a new product to get approval, superiority to existing therapies should be demonstrated. Getting an exhaustive view of the safety profile of a new medicinal product is usually not possible within clinical trials only, as rare adverse events may not become evident until a significant number of individuals are exposed to the drug. On the other hand, clinical trials can provide information regarding some common adverse events that might not have been detected post-marketing, the cardiovascular risk with COX-2 inhibitors observed in large randomised trials being a classical example [5].

\section{Changes in the European regulatory environment}

These general requirements still apply, but during the last decade there have been some major changes in the European regulatory system, with the overall aim of facilitating development and access to new treatments. The establishment of the European Medicines Agency (EMEA) in 1995, a network agency with contribution from all national authorities within the EU, marked the beginning of a new era. The EMEA was established mainly to help innovative biotechnology gain access to a wide market. For this, the centralised procedure for approval of hightechnology products was introduced, meaning that through one single procedure, marketing is possible in all EU countries simultaneously, following a positive opinion by the EMEA's scientific committees for human (CHMP) or veterinary medicinal products (CVMP), respectively and a subsequent Commission decision. The centralised procedure is mandatory for all high-technology products and for orphan medicinal products. The procedure is also mandatory for all new active substances within certain therapeutic areas, which from year 2008 will embrace: AIDS, oncology, neurodegenerative disorders, diabetes, autoimmune diseases and other immune dysfunctions and viral diseases [6].

In general, similar requirements apply for new medicinal products assessed through the centralised procedure, as for other applications, i.e., the benefit/risk (B/R) balance as regards quality, efficacy and safety of a new product has to be convincingly demonstrated in appropriate trials and in a sufficient number of patients. This may not always be possible, e.g., due to small populations with a certain disease. Furthermore, in some situations the medical need 
may be such that fast access to patients overrides the strict requirements. These particular situations are acknowledged within the European regulatory system. The orphan drug legislation and the paediatric legislation are intended to stimulate research and drug development in rare diseases and in children, respectively, by means of incentives to sponsors [7-10]. This concept has been developed further in the legislation for small and medium sized enterprises, intended to stimulate, through fee reductions, research and developments by these companies [11]. The conditional approval and approval under exceptional circumstances exist for products where there is a high medical need combined with difficulties in obtaining sufficient data, normally needed before marketing authorisation $[6,12]$. Conditional means that an approval is linked to certain conditions that have to be fulfilled post-marketing. However, prior to approval, the following conditions have to be met: demonstration of public health interest of product, including unmet medical need, and demonstration of positive $\mathrm{B} / \mathrm{R}$ balance based on evidence but pending completion of further studies. Such preliminary evidence could be based on results of interim analysis or surrogate endpoints (e.g., biomarkers or in a smaller population). Approval under exceptional circumstances applies when it is foreseen that normal data requirements are unlikely to be met and specific measures are thus usually needed postapproval, e.g., concerning safety of the product. The possibility for sponsors to request an accelerated assessment of innovative products of major interest is another way of gaining somewhat more rapid access to the market [6]. Thus, there are several situations were adaptations of the usual requirements for innovative therapies can be considered. These adaptations, however, may never compromise the safety of the patients. More rapid access to the market generally means reduced safety knowledge at the time of marketing authorisation and in this respect, well designed risk management/minimisation plans (RMP) are of utmost importance. Furthermore, implementation of these plans has to be ensured post-marketing. For the majority of centralised products, because of their innovative nature, a RMP is now a mandatory part of all new marketing authorisation applications [6].

\section{Registration of innovative therapies in Europe}

New technologies and emerging therapies, such as gene therapy, pharmacogenomics, proteomics and xenotransplantation are increasingly being submitted for marketing authorisation and challenge the current European regulatory system from a scientific, legal and regulatory perspective. Most of the emerging therapies aim to act as disease modifiers and a major benefit to public health is expected.
At the same time such therapies trigger new considerations for the safety as well as uncertainties about the applicability of diverse technical and scientific requirements. Several working groups under the CHMP are already dedicated to advanced therapies, such as the gene therapy WP, cell therapy WP and the pharmacogenetics WP. In 2008, the new regulation on advanced therapies and the establishment of the Committee for Advanced Therapies at the EMEA are expected to bring on board the much needed harmonisation with respect to advanced therapies [13].

\section{Future perspectives for registration of drugs in Europe}

The role and impact of the EMEA has successively grown since its establishment and the development has been fast. The original idea behind EMEA, to have access to and to harmonise the scientific resources within Europe, has been a successful one and the broad and extensive scientific competence available to the EMEA is reflected in the ever growing activities in different areas related to drug development, and not limited to approval of new therapies. EMEA's road map to year 2010 reflects well this extended activity but one of its key aspects is to encourage and facilitate innovation and research in the EU. At the same time, the Strategic Research Agenda, put together by the pharmaceutical industry in Europe, as a part of the Innovative Medicines Initiative, acknowledges the importance of regulatory authorities for drug development.

One of the major goals of the European Union is to build the most competitive and knowledge based economy in the world by 2010. In the Lisbon strategy for economic, social and environmental renewal, pharmaceutical and healthcare industries have a prominent role for the EU's economic competitiveness. The goal is to have by $2010,3 \%$ of Europe's GDP for investment in R\&D, achieved via one third from public sector and $2 / 3$ from private sector.

The Innovative Medicines Initiative derives from the Lisbon strategy. This initiative is funded by equal contribution from biopharmaceutical industry in Europe (EFPIA) and the European commission with 430 million euros each year for a period of 7 years in order to implement the recommendations proposed in its Strategic Research Agenda [14], prepared by the EFPIA.

This Strategic Research Agenda identifies several bottlenecks in drug development in the EU. One area for improvement relates to the prediction of clinical efficacy and safety for new products. For predicting efficacy, biomarkers have a prominent role. The acceptance of biomarkers or surrogate endpoints by regulatory authorities is for industry a major issue as this may facilitate and speed up clinical drug development programmes. Furthermore, some recent sensitive imaging techniques are seen as 
promising in the field of biomarkers [15]. There have been intensive interactions between all stakeholders on the issue of biomarkers, including workshops arranged by the EMEA with academia and health-care professionals and EFPIA, respectively. The discussions continue but currently, from a regulatory perspective, the use and validation of biomarkers in Phase II trials and for selective patient selection into clinical trials is endorsed, while for Phase III trials, only properly validated biomarkers can be accepted as surrogate endpoints, e.g., HbA1c [16]. Some biomarkers, such as bone mineral density (BMD), have been partly accepted for Phase III trials. While not accepted for initial applications for the indication of osteoporosis, BMD is accepted as a primary endpoint in Phase III bridging studies from postmenopausal women to men [17]. Biomarker validation, meaning that for certain biomarker there has to be confidence that changes in the marker reliably predicts the desired clinical endpoint, will always be critical for regulatory acceptance. Development and validation of biomarkers is resource demanding and will in the future need the combined efforts of academia, industry and regulatory authorities. Modelling and simulations are further tools that are increasingly being used in drug development, e.g., for dose selection [18]. Regulators support this approach, however, for efficacy and safety, modelling and simulations still cannot replace the required confirmatory evidence.

Another issue that is currently being discussed intensively by all stakeholders in drug development is a flexible approach in drug development e.g., in the design of clinical trials and statistical methods. The acceptance of adaptive methodologies with continuous assessment of data instead of the traditional separate Phase I to III trials can be expected to reduce the time span of clinical drug development and result in faster access of innovative therapies to patients. For the explorative phase of development, the use of adaptive designs is not controversial but for confirmatory trials, the use of adaptive designs needs further discussions. This is currently being addressed by the EMEA in collaboration with other stakeholders, including the development of a guideline [19] and a joint workshop with EMEA and industry on adaptive designs in confirmatory trials.

\section{Interaction between European regulatory authorities and pharmaceutical industry}

From the pharmaceutical industries perspective, interaction with European regulators during drug development and prior to regulatory approval is an issue for improvement. Currently, EMEA's interaction with pharmaceutical industry prior to marketing authorisation application has been mainly through its working parties dealing with innovative biological products, the Efficacy working party (EWP) and the Scientific advice working party (SAWP). Guideline development by the EWP is a transparent procedure with the possibility for interested parties to comment on draft guidelines during their development. The EMEA has prioritised guidelines in areas where there has been a need for a guideline. Currently, there seems to be a need, primarily for general guidelines on innovative statistical approaches as well as for tailored scientific guidance on quality and non-clinical issues, and this has been responded to, e.g., by the guideline on flexible design in confirmatory trials.

For issues where no guidance exists or in case companies wish to deviate from a given guideline, e.g., due to changing practises or new advances in science, it is recommended to seek scientific advice from the EMEA. Furthermore, as today's drug development increasingly moves towards targeted treatment solutions for patients with particular disease and particular molecular marker profile or otherwise defined disease pathology, a dialogue with regulators to discuss new approaches in the such targeted drug developments is important. The provision of scientific advice (and protocol assistance for orphan drugs) by the SAWP is an expanding activity of the EMEA. In 2006, the SAWP gave advice on 257 products in total compared with 58 in the year 2000 . This partly reflects the increased demand but also changes in the procedure for scientific advice implemented in 2006. The main changes were faster procedure and extended scope for scientific advice, including the possibility to obtain advice on RMP and conditional approval. Face-to-face discussion meetings during the process of giving advice are increasingly being used, but communication could be improved further. Increased possibilities for more informal discussions with the regulators are on the EMEA's agenda for the coming years, and already, less formal briefing meetings have been introduced by some of the EMEA's working parties. The need for a harmonised view, however, may limit the degree of flexibility as regards communication. The current procedure for provision of scientific advice involves not only two individual co-ordinators responsible for the advice but also there is extensive peer reviewing within the system, by other SAWP members and CHMP members. Furthermore, involvement of working parties in the finalisation of an advice has increased. This is bound to result in limitations with respect to the timeframe for providing advice. On the other hand this extensive peer reviewing should in the end result in a more robust advice facilitating assessment at the time of marketing authorisation application. Indeed, the central scientific advice provided by EMEA has proven to be a valuable tool in the development of innovative therapies [20]. For products intended for the 
centralised procedure, scientific advice by the EMEA should be obtained. For other products and for a less formal and less binding advice, and often a more rapid advice, the possibility of a national advice offered by many of the national regulatory authorities, remains. The EMEA encourages early dialogue on scientific and regulatory issues for new therapies, according to the general principle of life-cycle management of medicines. To this end the concept of "briefing meetings", informal advice given to companies willing to develop innovative biological products, has recently been introduced. Furthermore, an early dialogue provides opportunities for a more continuous and easier long-term dialogue. Formalisation of this concept, e.g., by introduction of systematic End-of-Phase II meetings is under discussion but is also a matter of resources. The alternative, to leave it up to companies whether or not to seek advice may in the end be more effective as the need for advice varies form one product to another.

\section{Interaction between European regulatory authorities and academia}

Interaction between regulatory authorities and the pharmaceutical industry can be regarded as relatively well established, but concerning academia there is room for improvement. Currently, EMEA has a list of over 3500 experts from different European countries [21] and from the beginning these experts have been involved in the scientific work performed at the EMEA. Further involvement of external experts has been established by the introduction of scientific advisory groups for important therapeutic areas.

Informal meetings, such as those organized by the Group for the Respect of Ethics and Excellence in Science (GREES) are another opportunity for discussion and exchange between regulators, academic scientists and representation of the pharmaceutical industry. They offer a non-official forum for debate on specific points of controversy. Their outcomes may be later taken into account by the relevant WP, if felt appropriate, at the time of the guidelines preparation [22]. Such interaction provided the basis for the 2006 revision of the "Guidelines on the Evaluation of Medicinal Products in the Treatment of Primary Osteoporosis" [17], see below.

Education and training are valuable tools in drug development and can be used to improve interaction between different stakeholders. The establishment of a European Medicines Research Academy for all professionals involved in biomedical research and development, including regulators, is an ambitious project for year 2013. This project aims at giving regular courses in pharmaceutical medicine at different universities in Europe. Such forum is an addition much welcomed to the currently limited (and uni-directional) interaction between scientists in academia, industry and regulatory authorities. All have a common goal and a common education and training will result in improved the communication during the process of bringing innovative therapies to the market, for the final benefit of the patients.

\section{Guidelines on the evaluation of medicinal products in the treatment of primary osteoporosis}

Following the original publication of the GREES [22], and extensive discussions at the CHMP, the therapeutic indication has been moved to the treatment of osteoporosis in postmenopausal at increased risk of fracture or, secondarily, the treatment of osteoporosis in men at increased risk of fracture. The new guideline document acknowledges that the risk of osteoporotic fracture is determined by several independent factors in addition to low bone mass. In order to encompass the complex relationship between bone mineral density (BMD), independent risk factors and the individual 10-year fracture risk, the suitable population for the clinical trials will now be postmenopausal women at increased risk of experiencing osteoporotic fractures, based on known skeletal independent risk factors such as age, BMD, prior fractures, a family history of hip fracture, high bone turnover, low body mass index, current tobacco use, and alcohol abuse, that result in an increased 10-year probability of fractures, regardless of the time elapsed since menopause. In men, epidemiological studies have shown a similar relationship between BMD and fracture risk in men and in postmenopausal women. However, since the other independent risk factors for fractures have not been as extensively validated in men as in women, it will be the applicant's responsibility to justify the criteria chosen for the inclusion of men in the pivotal study, including BMD, that will generate a fracture risk of a magnitude similar to that of postmenopausal osteoporotic women.

The applicant is requested to demonstrate the effect of the investigated medicinal product on both spinal and nonspinal fractures. For non-spinal fractures, either femoral (hip) or major non-vertebral (pelvis, distal femur, proximal tibia, ribs, proximal humerus, forearm, and hip) fractures should be assessed.

In principle, placebo-controlled trial should be performed whenever possible. However, if properly justified, non-inferiority trials versus active comparators could be considered if a clear justification of the margin of noninferiority is provided before the trial has started. The use of a placebo in diseases where an active treatment is available has been a matter of debate following the update of the Declaration of Helsinki by the World Medical Association [23] which questioned the trials design. A 
working group of the GREES [24] previously concluded that placebo-controlled trials remain the most efficient design to establish the efficacy and safety of a new agent for the treatment of postmenopausal osteoporosis. Such trials are feasible and ethically acceptable in patients with osteoporosis but without prevalent fractures. The new CHMP guideline document is in perfect accordance with these ethical concerns.

For compounds having demonstrated anti-fracture efficacy and for which the indication "treatment of osteoporosis in postmenopausal women at increased risk of fracture" has been previously granted for a specific dose, formulation or route of administration, an extension of the indication could be given for a new dose, route of administration or formulation on the basis of the demonstration of noninferiority in terms of BMD changes between the original and the new doses, formulations or routes of administration, in a study of minimum 1 year.

Whereas the gold standard for being granted a marketing authorization for the treatment of osteoporosis in men at increased risk of fracture remains the demonstration of antifracture efficacy, during a 2-year minimum, placebocontrolled, prospective study, once an initial marketing authorisation has been granted to a new chemical entity (NCE) for the treatment of postmenopausal osteoporosis in women at high risk of fracture, a separate bridging study of the same NCE, using the same formulation, dose and route of administration in male osteoporotic patients could be sufficient for being granted a marketing authorisation with the indication "treatment of osteoporosis in men at increased risk of fracture" providing conditions regarding the duration of the study, dosage, the magnitude of the fracture risk and the magnitude of the changes in BMD versus placebo are fulfilled.

Interestingly, an Experts panel representing the American Society for Bone and Mineral Research, the International Society for Clinical Densitometry and the National Osteoporosis Foundation (US) reached in 2008, conclusions which were quite concordant with the 2006 GREES document [25].

\section{Conclusion}

EMEA is a young agency that, since its establishment, has faced enormous challenges. Furthermore, the networking model has made it somewhat difficult for EMEA to elevate itself, but as the EMEA becomes more and more established, it becomes necessary to increase the public awareness and trust in the EMEA as a European and a world-leading regulatory agency. Communication and transparency are key words in this respect and do not only cover information, but also involvement of patients and health-care professionals in the work performed at the EMEA. This has already proven to be of great value, e.g., in the field of orphan products. The scientific basis for evaluation of products, however, sets limits to the degree patients can be involved in review processes. Health-care professional have to be made more aware of the impact of EMEA for medicines in Europe. All to often, the Food and Drug Administration (FDA) is being quoted by European health-care professionals as the only source regarding e.g., approvals of innovative therapies. In this respect, pharmaceutical industry in Europe as well as academia have a role in promoting the European regulatory perspectives of innovative therapies.

The fact that drug development is increasingly being done in a global environment is a challenge to the European regulatory system, e.g., as regards the acceptance of data from non-EU, and in particular Asian and third world countries. In this respect, enhanced collaboration with nonEU regulatory authorities is important. Interactions with the FDA are already established by the confidentiality arrangement on regulatory co-operation and transparency [26]. Internationally the EMEA contributes to the International Conference on Harmonisation (ICH) and collaborates with the World Health Organisation (WHO), e.g., by giving scientific opinions on products that are intended exclusively for markets outside Europe.

In conclusion, development of new, high quality, effective and safe medicines in Europe is the common goal of academia, pharmaceutical industry and regulatory authorities. To achieve this, it is important that regulatory requirements do not hinder innovation and vice versa, innovation cannot be allowed to proceed without concerns for public health. Interaction between stakeholders in pharmaceutical development is of the utmost importance. A dialogue has begun and in the future it will be the responsibility of all stakeholders to ensure continuous exchanges in an environment that is characterised by new scientific advances and global development programmes.

\section{Conflicts of Interest}

Name: Jean-Yves Reginster on behalf of the Department of Public Health, Epidemiology and Health Economics of the University of Liège, Liège, Belgium

Date: February 11, 2008

Consulting fees or paid advisory boards:

Servier, Novartis, Negma, Lilly, Wyeth, Amgen, GlaxoSmithKline, Roche, Merckle, Nycomed, NPS, Theramex.

Lecture fees when speaking at the invitation of a commercial sponsor: Merck Sharp and Dohme, Lilly, Rottapharm, IBSA, Genevrier, Novartis, Servier, Roche, GlaxoSmithKline, Teijin, Teva, Ebewee Pharma, Zodiac, Analis, Theramex, Nycomed, Novo-Nordisk.

Grant Support from Industry:

Bristol Myers Squibb, Merck Sharp \& Dohme, Rottapharm, Teva, Lilly, Novartis, Roche, GlaxoSmithKline, Amgen, Servier. 


\section{References}

1. Directive 2001/20/EC of 4 April 2001 on the approximation of the laws, regulations and administrative provisions of the Member States relating to the implementation of good clinical practice in the conduct of clinical trials on medicinal products for human use (Official Journal L 121, 1/5/2001 p. 34-44). http://ec.europa.eu/ enterprise/pharmaceuticals/eudralex/

2. Commission Directive 2005/28/EC of 8 April 2005 laying down principles and detailed guidelines for good clinical practice as regards investigational medicinal products for human use, as well as the requirements for authorisation of the manufacturing or importation of such products (Official Journal L 91, 9/4/2005 p. 1319). http://ec.europa.eu/enterprise/pharmaceuticals/eudralex/

3. Commission Directive 2003/94/EC of 8 October 2003 laying down the principle and guidelines of good manufacturing practice in respect of medicinal products for human use and investigational medicinal products for human use (Official Journal L 262, 14/10/2003 p. 2226). http://ec.europa.eu/enterprise/pharmaceuticals/eudralex/

4. Reginster JY (2007) The efficacy of glucosamine sulfate in osteoarthritis: financial and nonfinancial conflict of interest. Arthritis Rheum 56:2005-2010

5. Bresalier RS (2005) Cardiovascular events associated with rofecoxib in a colorectal adenoma chemoprevention trial. NEJM 352:1092-1102

6. Regulation (EC) No 726/2004 of 31 March 2004 laying down Community procedures for the authorisation and supervision of medicinal products for human and veterinary use and establishing a European Medicines Agency (Official Journal L 136, 30/4/2004 p. 1-24). http://ec.europa.eu/enterprise/pharmaceuticals/eudralex/

7. Regulation (EC) No 141/2000 of 16 December 1999 on orphan medicinal products (Official Journal L 18, 22/1/2000 p. 1 - 5). http://ec.europa.eu/enterprise/pharmaceuticals/eudralex/

8. Regulation (EC) No 847/2000 of 27 April 2000 laying down the provisions for implementation of the criteria for designation of a medicinal product as an orphan medicinal product and definitions of the concepts 'similar medicinal product' and' clinical superiority' (Official Journal L 103, 28/4/2000 p. 5 - 8). http://ec. europa.eu/enterprise/pharmaceuticals/eudralex/

9. Regulation (EC) No 1901/2006 of 12 December 2006 on medicinal products for paediatric use and amending Regulation (EEC) No 1768/92, Directive 2001/20/EC, Directive 2001/83/EC and Regulation (EC) No 726/2004 (Official Journal L 378, 27/12/2006 p. 20-21). http://ec.europa.eu/enterprise/pharmaceuticals/eudralex/

10. Regulation (EC) No 1905/2005, of 14 November 2005, amending Regulation (EC) No $297 / 95$ on fees payable to the European Medicines Agency (Official Journal L 340, 13/11/2005 p. 1 - 9). http://ec.europa.eu/enterprise/pharmaceuticals/eudralex/

11. Regulation (EC) No 2049/2005, of 15 December 2005, laying down, pursuant to Regulation (EC) No 726/2004 of the European Parliament and of the Council, rules regarding the payment of fees to, and the receipt of administrative assistance from, the European Medicines Agency by micro, small and medium-sized enterprises (Official Journal L 329, 16/12/2005 p. 4 - 7). http://ec.europa.eu/ enterprise/pharmaceuticals/eudralex/

12. Regulation (EC) No 507/2006 of 29 March 2006 on the conditional marketing authorisation for medicinal products for human use falling within the scope of Regulation (EC) No 726/
2004 of the European Parliament and of the Council (Official Journal L 92, 30/3/2006 p. 6 - 9). http://ec.europa.eu/enterprise/ pharmaceuticals/eudralex/

13. Medicines and Emerging Science-Advanced Therapies http:// www.emea.europa.eu/htms/human/mes/advancedtherapies.htm

14. Health and the EU Lisbon Agenda http://www.efpia.org/6_publ/ Healthlisbonagenda.pdf

15. Abadie E, Ethgen D, Avouac B, et al. (2004) Recommendations for the use of new methods to assess the efficacy of diseasemodifying drugs in the treatment of osteoarthritis. Osteoarthr Cartil 12:263-268

16. Note for Guidance on Clinical Investigation of Medicinal Products in the Treatment of Diabetes Mellitus CPMP/EWP/1080/00. http://www.emea.europa.eu/pdfs/human/ewp/108000cn.pdf

17. Guideline on the Evaluation of Medicinal Products in the Treatment of Primary Osteoporosis. CPMP/EWP/552/95. http:// www.emea.europa.eu/pdfs/human/ewp/55295enfin.pdf

18. Reginster JY, Gieschke R (2006) Clinical utility of a pharmacostatistical model for ibandronate in postmenopausal osteoporosis. Curr Drug Metab 7:827-836

19. Reflection Paper on Methodological issues in confirmatory clinical trials with flexible design and analysis plan. CHMP/ EWP/2459/02 http://www.emea.europa.eu/pdfs/human/ewp/ $245902 \mathrm{cn} . \mathrm{pdf}$

20. Survey 2006 on the performance of EMEA scientific procedures for medicinal products for human use (EMEA/489472/2006). http://www.emea.europa.eu/pdfs/general/direct/48948206en.pdf

21. Committee for Medicinal Products for Human Use (CHMP) CHMP Members http:/www.emea.europa.eu/htms/general/contacts/ CHMP/CHMP_members.html

22. Reginster JY, Abadie E, Delmas PD, Rizzoli R, Dere W, der Auwera P, Avouac B, Brandi ML, Daifotis A, Diez-Perez A, Calvo G, Johnell O, Kaufman JM, Kreutz G, Laslop A, Lekkerkerker F, Mitlak B, Nilsson P, Orloff J, Smillie M, Taylor A, Tsouderos Y, Ethgen D, Flamion B (2006) Recommendations for an update of the current (2001) regulatory requirements for registration of drugs to be used in the treatment of osteoporosis in postmenopausal women and in men. Osteoporos Int 17:1-7

23. World Medical Association declaration of Helsinki (1997) Recommendation guiding physician and biochemical research involving human subjects. JAMA 277:225-226

24. Delmas PD, Calvo G, Boers M, Abadie E, Avouac B, Kahan A, Kaufman JM, Laslop A, Lekkerkerker JF, Nilsson P, Van ZwietenBoot B, Kreutz G, Reginster JY; Group for the Respect of Ethics and Excellence in Science (GREES) (2002) The use of placebocontrolled and non-inferiority trials for the evaluation of new drugs in the treatment of postmenopausal osteoporosis. Osteoporos Int 13:1-5

25. Silverman SL, Cummings SR, Watts NB; for the Consensus Panel of the ASBMR, ISCD, and NOF (2008) Recommendations for the Clinical Evaluation of Agents for Treatment of Osteoporosis: Consensus of an Expert Panel Representing the American Society for Bone and Mineral Research (ASBMR), the International Society for Clinical Densitometry (ISCD), and the National Osteoporosis Foundation (NOF). J Bone Miner Res 23:159-165

26. Confidentiality Arrangements concluded between the EU (EC and EMEA) and the US FDA/DHHS Implementation Plan for Medicinal Products for Human Use. http://www.emea.europa.eu/ $\mathrm{pdfs} /$ general/direct/pr/confidentiality.pdf 